\title{
Hierarchies of equations for the Legendre transforms of energy functionals
}

\author{
Á. Nagy \\ Institute of Theoretical Physics, Kossuth Lajos University, H-4010 Debrecen, Hungary
}

(Received 18 January 1994; revised manuscript received 4 January 1995)

\begin{abstract}
Hierarchies of equations have been derived for the Legendre transform of the energy functionals of the density-functional theory. As an application, the Thomas-Fermi and the Thomas-Fermi-Dirac models are recovered and the Pauli energy is discussed. Useful relations for the electron density in the weighted-density and gradient approximations are derived.
\end{abstract}

PACS number(s): 31.20.Sy, 71.10. $+\mathrm{x}, 05.30 . \mathrm{Fk}$

\section{INTRODUCTION}

The development and improvement of approximations to energy functionals have been major subjects of research in density-functional theory [1]. Nevertheless, the exact form of the kinetic, the exchange, and the correlation energy functionals is still unknown. For development of approximations, exact relations and criteria that are fulfilled by the exact functionals are of great help. Recently, Levy [2] showed that coordinate scaling is a powerful tool in constructing new approximate functionals. In a previous paper, exact hierarchies of equations [3] have been derived for the density-functional theory. Here, hierarchies of equations are presented for the Legendre transforms of the energy functionals. These hierarchies of equations for the energy functionals are also criteria to be fulfilled by the functionals. These equations can be applied to construct new functionals.

The main results of this paper are as follows: the first,

$$
\begin{aligned}
3 n+\vec{r} \cdot \vec{\nabla} n= & -\int d \vec{r}_{1} u\left(\vec{r}_{1}\right)\left(2+\vec{r}_{1} \cdot \vec{\nabla}_{1}\right) \frac{\delta n\left(\vec{r}_{1}\right)}{\delta u(\vec{r})} \\
& -\frac{\delta T}{\delta u(\vec{r})},
\end{aligned}
$$

the second,

$$
\begin{aligned}
&\left(5+\vec{r} \cdot \vec{\nabla}+\vec{r}_{1} \cdot \vec{\nabla}_{1}\right) \frac{\delta n(\vec{r})}{\delta u\left(\vec{r}_{1}\right)} \\
&=-\int d \vec{r}_{2} u\left(\vec{r}_{2}\right)\left(2+\vec{r}_{2} \cdot \vec{\nabla}_{2}\right) \frac{\delta^{2} n\left(\vec{r}_{2}\right)}{\delta u(\vec{r}) \delta u\left(\vec{r}_{1}\right)} \\
&-\frac{\delta^{2} T}{\delta u(\vec{r}) \delta u\left(\vec{r}_{1}\right)},
\end{aligned}
$$

and higher-order equations of the hierarchy for the Legendre transform of the total energy; the first,

$$
3 n+\vec{r} \cdot \vec{\nabla} n=-\int d \vec{r}_{1}\left(1+\vec{r}_{1} \cdot \vec{\nabla}_{1}\right) \frac{\delta n\left(\vec{r}_{1}\right)}{\delta \widetilde{u}(\vec{r})},
$$

the second,

$$
\begin{aligned}
\left(4+\vec{r} \cdot \vec{\nabla}+\vec{r}_{1} \cdot \vec{\nabla}_{1}\right) \frac{\delta n(\vec{r})}{\delta \tilde{u}\left(\vec{r}_{1}\right)} \\
\quad=-\int d \vec{r}_{2} \widetilde{u}\left(\vec{r}_{2}\right)\left(1+\vec{r}_{2} \cdot \vec{\nabla}_{2}\right) \delta^{2} n\left(\vec{r}_{2}\right) \frac{\delta^{2} n\left(\vec{r}_{2}\right)}{\delta \widetilde{u}(\vec{r}) \delta \widetilde{u}\left(\vec{r}_{1}\right)},
\end{aligned}
$$

and higher-order equations of the kinetic-energy functional; the first,

$$
3 n+\vec{r} \cdot \vec{\nabla} n=-\int d \vec{r}_{1} v_{x}\left(\vec{r}_{1}\right)\left(2+\vec{r}_{1} \cdot \vec{\nabla}_{1}\right) \frac{\delta n\left(\vec{r}_{1}\right)}{\delta v_{x}(\vec{r})},
$$

the second,

$$
\begin{aligned}
(5+\vec{r} \cdot \vec{\nabla} & \left.+\vec{r}_{1} \cdot \vec{\nabla}_{1}\right) \frac{\delta n(\vec{r})}{\delta v_{x}\left(\vec{r}_{1}\right)} \\
& =-\int d \vec{r}_{2} v_{x}\left(\vec{r}_{2}\right)\left(2+\vec{r}_{2} \cdot \vec{\nabla}_{2}\right) \frac{\delta^{2} n\left(\vec{r}_{2}\right)}{\delta v_{x}(\vec{r}) \delta v_{x}\left(\vec{r}_{1}\right)},
\end{aligned}
$$

and higher-order equations of the hierarchy for the Legendre transform of the exchange energy functional. $n, v_{x}$, and $T$ are the electron density, the exchange potential, and the kinetic energy, respectively. Moreover,

$$
u(\vec{r})=v(\vec{r})-\mu
$$

and

$$
\widetilde{u}(\vec{r})=v_{\mathrm{KS}}(\vec{r})-\mu,
$$

where $v, v_{\mathrm{KS}}$, and $\mu$ are the external potential, the KohnSham potential, and the chemical potential, respectively.

The hierarchies of equations can be used to check the quality of approximate functionals. One can use first the zeroth equations of the hierarchies, such as Eq. (39) or Eq. (44) and Eq. (59) or Eq. (62). If one inserts the approximating expressions into both sides of the zeroth equation, the values of two integrals, i.e., two numbers, can be compared and used for the checking. However, if the first equations of the hierarchies are applied as criteria for the approximating functionals, such as Eqs. (1), (3), or (5), the comparison of the two sides of these equations compares two functions of $\vec{r}$ instead of two numbers. So the first equations of the hierarchy give more information. In addition, higher-order equations of the hierarchies provide even more information. Certainly, to 
apply these equations, higher-order functional derivatives of the functionals are needed.

In a previous paper [3], hierarchies of equations for the energy functionals were presented. Here, hierarchies of equations for the Legendre transforms of energy functionals have been derived. These hierarchies are closely related, though there are considerable differences. Whereas in [3], the energy functionals were functionals of the electron density, here the Legendre transforms of energy functionals as functionals of potentials are presented. The electron density appears as a functional derivative of Legendre transforms, and the first-order equations of the hierarchies provide equations for the electron density. Whereas in [3], in the hierarchy equations, the hardness kernel [4] had a very important role, in these new equations the softness kernel [5] is the fundamental quantity. These newly derived hierarchies provide explicit equations for the electron density and its functional derivatives. These comparisons may reveal interesting connections between quantities of physical interest. They present alternative ways of deriving new approximate relations. There may be problems where it is more natural or convenient to apply these equations. For example, as is shown in Sec. VIII, the Legendre transform of the kinetic energy is especially suitable for studying the Pauli energy.

The Legendre transform of the exchange energy is studied in the weighted-density and gradient approximations. The first equation of the hierarchy gives a useful differential equation for the electron density. This equation can be used in constructing or improving approximating functionals.

\section{SOFTNESS KERNEL AND THE LOCAL SOFTNESS}

The ground-state energy of a system with $N$ interacting electrons in an external field $v(\vec{r})$ is

$$
E[n]=\int d \vec{r} n(\vec{r}) v(\vec{r})+F[n],
$$

where

$$
F[n]=T[n]+V_{e e}[n] .
$$

$T$ and $V_{e e}$ are the kinetic and the electron-electron energy functionals of the electron density $n$. Let us consider $\Omega$ defined as the functional Legendre transform of $F[n]$ :

$$
\Omega[u]=F[n]+\int d \vec{r} n(\vec{r}) u(\vec{r}) .
$$

The functional derivative of $\Omega$ with respect to $u$ yields the density

$$
\left.\frac{d \Omega[n]}{\delta u(\vec{r})}\right|_{n}=n(\vec{r} ; u)
$$

The second functional derivate of $\Omega$ can be given by the so-called softness kernel $s\left(\vec{r}, \vec{r}^{\prime} ; u\right)[5]$

$$
\left.\frac{\delta^{2} \Omega[u]}{\delta u(\vec{r}) \delta u\left(\vec{r}^{\prime}\right)}\right|_{n}=\frac{\delta n(\vec{r} ; u)}{\delta u\left(\vec{r}^{\prime}\right)}=-s\left(\vec{r}, \vec{r}^{\prime} ; u\right),
$$

and the third functional derivative is

$$
\begin{aligned}
\left.\frac{\delta^{3} \Omega[u]}{\delta u(\vec{r}) \delta u\left(\vec{r}^{\prime}\right) \delta u\left(\vec{r}^{\prime \prime}\right)}\right|_{n} & =\left.\frac{\delta^{2} n(\vec{r}, u)}{\delta u\left(\vec{r}^{\prime}\right) \delta u\left(\vec{r}^{\prime \prime}\right)}\right|_{n} \\
& =-\left.\frac{\delta s\left(\vec{r}, \vec{r}^{\prime} ; u\right)}{\delta u\left(\vec{r}^{\prime \prime}\right)}\right|_{n} \\
& =-s\left(\vec{r}, \vec{r}^{\prime}, \vec{r}^{\prime \prime} ; u\right) .
\end{aligned}
$$

From the identity

$$
\int \frac{\delta n(\vec{r})}{\delta u\left(\vec{r}^{\prime \prime}\right)} \frac{\delta u\left(\vec{r}^{\prime \prime}\right)}{\delta n\left(\vec{r}^{\prime}\right)} d \vec{r}^{\prime \prime}=\delta\left(\vec{r}-\vec{r}^{\prime}\right),
$$

we get

$$
\int d \vec{r}^{\prime \prime} s\left(\vec{r}, \vec{r}^{\prime \prime} ; u\right) \eta\left(\vec{r}^{\prime \prime}, \vec{r}^{\prime} ; n\right)=\delta\left(\vec{r}-\vec{r}^{\prime}\right) ;
$$

that is, the hardness kernel $\boldsymbol{\eta}$ is the inverse of the softness kernel.

Multiplying Eq. (16) by $n\left(\vec{r}^{\prime \prime}\right)$ and integrating over $\vec{r}^{\prime \prime}$, we get

$$
\int s\left(\vec{r}, \vec{r}^{\prime}\right) \eta\left(\vec{r}^{\prime}\right) d \vec{r}^{\prime}=\frac{1}{N} n(\vec{r}),
$$

with the definition of the local hardness:

$$
\eta(\vec{r})=\frac{1}{N} \int \eta\left(\vec{r}, \vec{r}^{\prime}\right) n\left(\vec{r}^{\prime}\right) d \vec{r}^{\prime}
$$

Integrating Eq. (17) over $\vec{r}$, we find that the local hardness and softness are inverses in the sense that

$$
\int s(\vec{r}) \eta(\vec{r}) d \vec{r}=1
$$

where the local softness is

$$
s(\vec{r})=\int d \vec{r}^{\prime} s\left(\vec{r}, \vec{r}^{\prime} ; u\right) .
$$

From Eq. (17) it follows that the relation between the partial derivative $\partial /\left.\partial \mu\right|_{u}$ and the functional derivative $\delta /\left.\delta u(\vec{r})\right|_{n}$ is

$$
\left.\frac{\partial}{\partial \mu}\right|_{u}=-\left.\int d \vec{r} \frac{\delta}{\delta u(\vec{r})}\right|_{n} .
$$

Applying this operation to the density $n$ and the functional $\Omega$, we get

$$
\left.\frac{\partial n(\vec{r})}{\partial \mu}\right|_{u}=-\left.\int d \vec{r}^{\prime} \frac{\delta n(\vec{r})}{\delta u\left(\vec{r}^{\prime}\right)}\right|_{n}=\int d \vec{r}^{\prime} s\left(\vec{r}, \vec{r}^{\prime}\right)=s(\vec{r})
$$

and

$$
\left.\frac{\partial \Omega}{\partial \mu}\right|_{u}=-\int d \vec{r} n(\vec{r}, u)=-N .
$$

Another application of Eq. (21) to Eq. (23) gives the global softness $S$

$$
\left.\frac{\partial^{2} \Omega}{\partial \mu^{2}}\right|_{u}=-\int d \vec{r} d \vec{r}^{\prime} s\left(\vec{r}, \vec{r}^{\prime}\right)=-\left.\frac{\partial N}{\partial \mu}\right|_{u}=-S=-\frac{1}{\eta}
$$

or the inverse of global hardness $\eta$.

The functional derivative of the chemical potential can 
also be considered. As

$$
\mu=\left(\frac{\partial E}{\partial N}\right]_{u},
$$

we obtain

$$
\left.\frac{\delta \mu}{\delta u(\vec{r})}\right|_{N}=\frac{\partial}{\partial N}\left[\frac{\delta E}{\delta u(\vec{r})}\right]_{n}=\left.\frac{\partial n(\vec{r})}{\partial N}\right|_{u}=f(\vec{r}),
$$

because

$$
E=F[n]+\int d \vec{r} n(\vec{r})[u(\vec{r})+\mu]
$$

and $f(\vec{r})$ is the Fukui function. Equations (7) and (26) lead to

$$
\left.\int \frac{\delta \mu}{\delta u(\vec{r})}\right|_{N} d \vec{r}=\int f(\vec{r}) d \vec{r}=1 .
$$

The identity

$$
\left.\frac{\partial n(\vec{r})}{\partial \mu}\right|_{u}=\left.\frac{\partial n(\vec{r})}{\partial N} \frac{\partial N}{\partial \mu}\right|_{u}
$$

gives the well-known relation between the local softness $s(\vec{r})$, the global softness $S$, and the Fukui function $f(\vec{r})$ $[6,7]$ :

$$
s(\vec{r})=S f(\vec{r}) .
$$

\section{HIERARCHY OF EQUATIONS FOR THE LEGENDRE OF TRANSFORM OF THE TOTAL ENERGY FUNCTIONAL}

The starting point of the derivation of the hierarchy equations is the virial relation of Levy and Perdew [8]:

$$
F[n]+T[n]=-\int d \vec{r} n(\vec{r}) \vec{r} \cdot \vec{\nabla} \frac{\delta F[n]}{\delta n(\vec{r})} .
$$

From the Hohenberg-Kohn theorem, the Euler-Lagrange equation

$$
\frac{\delta F[n]}{\delta n(\vec{r})}+v(\vec{r})=\mu
$$

follows. Taking the gradient of the equation we arrive at

$$
\vec{\nabla} \frac{\delta F[n]}{\delta n(\vec{r})}=\vec{\nabla}[\mu-v(\vec{r})]=-\vec{\nabla} u(\vec{r})
$$

Using Eqs. (11) and (33), the Levy-Perdew relation has the form

$$
\Omega[u]+T[n]-\int d \vec{r} n(\vec{r}) u(\vec{r})=\int d \vec{r} n(\vec{r}) \vec{r} \cdot \vec{\nabla} u(\vec{r}),
$$

or after partially integrating the right-hand side of this equation,

$$
\begin{aligned}
\Omega[u]=-2 \int d \vec{r} n(\vec{r}) & u(\vec{r}) \\
& -\int d \vec{r} u(\vec{r}) \vec{r} \cdot \vec{\nabla} n(\vec{r})-T[n] .
\end{aligned}
$$

The functional differentiation of Eq. (35) with respect to $u(\vec{r})$ gives

$$
\begin{aligned}
\frac{\delta \Omega}{\delta u(\vec{r})}=n(\vec{r})= & -2 n(\vec{r})-\vec{r} \cdot \vec{\nabla} n(\vec{r}) \\
& -2 \int d \vec{r}_{1} u\left(\vec{r}_{1}\right) \frac{\delta n\left(\vec{r}_{1}\right)}{\delta u(\vec{r})} \\
& -\int d \vec{r}_{1} u\left(\vec{r}_{1}\right) \vec{r}_{1} \cdot \vec{\nabla}_{1} \frac{\delta n\left(\vec{r}_{1}\right)}{\delta u(\vec{r})}-\frac{\delta T}{\delta u(\vec{r})},
\end{aligned}
$$

or with Eq. (13),

$$
3 n+\vec{r} \cdot \vec{\nabla} n=\int d \vec{r}_{1} u\left(\vec{r}_{1}\right)\left(2+\vec{r}_{1} \cdot \vec{\nabla}_{1}\right) s\left(\vec{r}, \vec{r}_{1}\right)-\frac{\delta T}{\delta u(\vec{r})},
$$

which is the first equation of the hierarchy. The functional derivation of Eq. (37) with respect to $u$ provides the second equation of the hierarchy:

$$
\begin{aligned}
& 5 s\left(\vec{r}, \vec{r}_{1}\right)+\left(\vec{r} \cdot \vec{\nabla}+\vec{r}_{1} \cdot \vec{\nabla}_{1}\right) s\left(\vec{r}, \vec{r}_{1}\right) \\
&=-\int d \vec{r}_{2} u\left(\vec{r}_{2}\right)\left(2+\vec{r}_{2} \cdot \vec{\nabla}_{2}\right) s\left(\vec{r}, \vec{r}_{1}, \vec{r}_{2}\right) \\
&+\frac{\delta^{2} T}{\delta u(\vec{r}) \delta u\left(\vec{r}_{1}\right)} .
\end{aligned}
$$

Further derivation will lead to the higher-order equations of the hierarchy. In passing we remark that Parr and Gázquez [9] called the negative of $\Omega$ the hardness functional. However, the hardness functional is a functional of the density, whereas $\Omega$ is a functional of $u$.

\section{HIERARCHY OF EQUATIONS \\ FOR THE LEGENDRE TRANSFORM OF THE KINETIC ENERGY FUNCTIONAL}

The first hierarchy of equations for the noninteracting kinetic energy was derived by Kugler [10]. The noninteracting kinetic energy can be written as a virial relation:

$$
T_{s}[n]=\frac{1}{2} \int d \vec{r} n(\vec{r}) \vec{r} \cdot \vec{\nabla} v_{\mathrm{KS}},
$$

where $v_{\mathrm{KS}}$ is the Kohn-Sham potential. The EulerLagrange equation can also be written as

$$
\frac{\delta T_{s}[n]}{\delta n(\vec{r})}+v_{\mathrm{Ks}}-\mu=0 .
$$

Combining Eqs. (39) and (40) we get

$$
T_{s}[n]=-\frac{1}{2} \int d \vec{r} n(\vec{r}) \vec{r} \cdot \vec{\nabla} \frac{\delta T_{s}[n]}{\delta n(\vec{r})} .
$$

The functional Legendre transform of the noninteracting kinetic energy $T_{s}$ is

$$
Q_{s}[\tilde{u}]=T_{s}[n]+\int d \vec{r} n(\vec{r}) \tilde{u}(\vec{r}),
$$

where

$$
\widetilde{u}=v_{\mathrm{KS}}-\mu .
$$


Using Eqs. (40)-(43) we arrive at the zeroth equation of the hierarchy of the kinetic energy:

$$
Q_{s}[\tilde{u}]=\int d \vec{r} n(\vec{r}) \tilde{u}(\vec{r})+\frac{1}{2} \int d \vec{r} n(\vec{r}) \vec{r} \cdot \vec{\nabla} \widetilde{u}(\vec{r}),
$$

or in another form

$$
Q_{s}[\widetilde{u}]=-\frac{1}{2} \int d \vec{r} \widetilde{u}(\vec{r})(1+\vec{r} \cdot \vec{\nabla}) \frac{\delta Q_{s}}{\delta \widetilde{u}(\vec{r})}
$$

From Eq. (42),

$$
\frac{\delta Q_{s}[\tilde{u}]}{\delta \tilde{u}(\vec{r})}=n(\vec{r}) .
$$

Functional differentiation with respect to $\widetilde{u}(\vec{r})$ of Eq. (45) leads to the first equation of the hierarchy of the noninteracting kinetic energy:

$$
3 n+\vec{r} \cdot \vec{\nabla} n=\int d \vec{r}_{1} \tilde{u}\left(\vec{r}_{1}\right)\left(1+\vec{r}_{1} \cdot \vec{\nabla}_{1}\right) s_{Q}\left(\vec{r}, \vec{r}_{1}\right),
$$

where

$$
s_{Q}\left(\vec{r}, \vec{r}_{1}\right)=-\frac{\delta n(\vec{r})}{\delta \tilde{u}\left(\vec{r}_{1}\right)}=-\frac{\delta^{2} Q_{s}[\tilde{u}]}{\delta \tilde{u}(\vec{r}) \delta \tilde{u}\left(\vec{r}_{1}\right)}
$$

is the noninteracting kinetic softness kernel. Functionally differentiating Eq. (47), we obtain the second equation of the hierarchy:

$$
\begin{aligned}
4 s_{Q}\left(\vec{r}, \vec{r}_{1}\right)+ & \left(\vec{r} \cdot \vec{\nabla}+\vec{r}_{1} \cdot \vec{\nabla}_{1}\right) s_{Q}\left(\vec{r}, \vec{r}_{1}\right) \\
& =-\int d \vec{r}_{2} \widetilde{u}\left(\vec{r}_{2}\right)\left(1+\vec{r}_{2} \cdot \vec{\nabla}_{2}\right) s_{Q}\left(\vec{r}, \vec{r}_{1}, \vec{r}_{2}\right)
\end{aligned}
$$

Higher-order equations can be similarly derived.

\section{HIERARCHY OF EQUATIONS FOR THE LEGENDRE TRANSFORM OF THE EXCHANGE-CORRELATION ENERGY FUNCTIONAL}

The Levy-Perdew relation for the exchange-correlation energy has the form [8]

$$
E_{\mathrm{xc}}[n]+T_{c}[n]=-\int d \vec{r} n(\vec{r}) \vec{r} \cdot \vec{\nabla} v_{\mathrm{xc}}(\vec{r}),
$$

where

$$
v_{\mathrm{xc}}(\vec{r})=\frac{\delta E_{\mathrm{xc}}[n]}{\delta n(\vec{r})}
$$

is the exchange-correlation potential. Let $P\left[v_{\mathrm{xc}}\right]$ be defined as the functional Legendre transform of the exchange-correlation energy $E_{\mathrm{xc}}$ :

$$
P\left[v_{\mathrm{xc}}\right]=E_{\mathrm{xc}}[n]-\int d \vec{r} n(\vec{r}) v_{\mathrm{xc}}(\vec{r}) .
$$

Using Eqs. (50) and (52) we obtain the zeroth order of the hierarchy for the exchange-correlation energy:

$$
P\left[v_{\mathrm{xc}}\right]=\int d \vec{r} v_{\mathrm{xc}}(\vec{r})(2+\vec{r} \cdot \vec{\nabla}) n(\vec{r})-T_{c} .
$$

From Eq. (52),

$$
\frac{\delta P\left[v_{\mathrm{xc}}\right]}{\delta v_{\mathrm{xc}}(\vec{r})}=-n(\vec{r}) \text {. }
$$

The functional differentiation with respect to $v_{\mathrm{xc}}(\vec{r})$ of Eq. (53) leads to the first equation of the hierarchy,

$$
3 n+\vec{r} \cdot \vec{\nabla} n=\int d \vec{r}_{1} v_{\mathrm{xc}}\left(\vec{r}_{1}\right)\left(2+\vec{r}_{1} \cdot \vec{\nabla}_{1}\right) s_{\mathrm{xc}}\left(\vec{r}, \vec{r}_{1}\right)+\frac{\delta T_{c}}{\delta v_{\mathrm{xc}}},
$$

while the second functional differentiation provides the second equation of the hierarchy,

$$
\begin{aligned}
5 s_{\mathrm{xc}}\left(\vec{r}, \vec{r}_{1}\right)+(\vec{r} \cdot \vec{\nabla} & \left.+\vec{r}_{1} \cdot \vec{\nabla}_{1}\right) s_{\mathrm{xc}}\left(\vec{r}, \vec{r}_{1}\right) \\
& =-\int d \vec{r}_{2} v_{\mathrm{xc}}\left(\vec{r}_{2}\right)\left(2+\vec{r}_{2} \cdot \vec{\nabla}_{2}\right) s_{\mathrm{xc}}\left(\vec{r}, \vec{r}_{1}, \vec{r}_{2}\right) \\
& -\frac{\delta^{2} T_{c}}{\delta v_{\mathrm{xc}}(\vec{r}) \delta v_{\mathrm{xc}}\left(\vec{r}_{1}\right)} .
\end{aligned}
$$

The exchange-correlation softness kernels are defined as

$$
s_{\mathrm{xc}}\left(\vec{r}, \vec{r}_{1}\right)=-\frac{\delta n(\vec{r})}{\delta v_{\mathrm{xc}}\left(\vec{r}_{1}\right)}=-\frac{\delta^{2} P_{\mathrm{xc}}}{\delta v_{\mathrm{xc}}(\vec{r}) \delta v_{\mathrm{xc}}\left(\vec{r}_{1}\right)}
$$

and

$$
\begin{aligned}
s_{\mathrm{xc}}\left(\vec{r}, \vec{r}_{1}, \vec{r}_{2}\right) & =-\frac{\delta^{2} n(\vec{r})}{\delta v_{\mathrm{xc}}(\vec{r}) \delta v_{\mathrm{xc}}\left(\vec{r}_{1}\right)} \\
& =-\frac{\delta^{2} P_{\mathrm{xc}}}{\delta v_{\mathrm{xc}}(\vec{r}) \delta v_{\mathrm{xc}}\left(\vec{r}_{1}\right) \delta v_{\mathrm{xc}}\left(\vec{r}_{2}\right)} .
\end{aligned}
$$

Higher-order equations can also be derived.

\section{HIERARCHY OF EQUATIONS FOR THE LEGENDRE TRANSFORM OF THE EXCHANGE ENERGY FUNCTIONAL}

To derive the hierarchy equations for the exchange energy, we rewrite the Levy-Perdew relation [8] for the exchange energy,

$$
E_{x}[n]=-\int d \vec{r} n(\vec{r}) \vec{r} \cdot \vec{\nabla} v_{x}(\vec{r}),
$$

with the Legendre transform of $E_{x}$,

$$
P_{x}\left[v_{x}\right]=E_{x}[n]-\int d \vec{r} n(\vec{r}) v_{x}(\vec{r})
$$

The exchange potential $v_{x}(\vec{r})$ is given by

$$
v_{x}(\vec{r})=\frac{\delta E_{x}[n]}{\delta n(\vec{r})}
$$

From Eqs. (59) and (60) the zeroth-order equation of the hierarchy,

$$
P_{x}\left[v_{x}\right]=\int d \vec{r} v_{x}(\vec{r})[2+\vec{r} \cdot \vec{\nabla}] n(\vec{r}),
$$

is obtained. The first and second functional derivatives of Eq. (62) lead to the first,

$$
3 n+\vec{r} \cdot \vec{\nabla} n=\int d \vec{r}_{1} v_{x}\left(\vec{r}_{1}\right)\left(2+\vec{r}_{1} \cdot \vec{\nabla}_{1}\right) s_{x}\left(\vec{r}, \vec{r}_{1}\right),
$$

and the second equations of the hierarchy, 


$$
\begin{aligned}
5 s_{x}\left(\vec{r}, \vec{r}_{1}\right)+\left(\vec{r} \cdot \vec{\nabla}+\vec{r}_{1} \cdot \vec{\nabla}_{1}\right) s_{x}\left(\vec{r}, \vec{r}_{1}\right) \\
\quad=\int d \vec{r}_{2} v_{x}\left(\vec{r}_{2}\right)\left[2+\vec{r}_{2} \cdot \vec{\nabla}_{2}\right] s_{x}\left(\vec{r}, \vec{r}_{1}, \vec{r}_{2}\right),
\end{aligned}
$$

where

$$
s_{x}\left(\vec{r}, \vec{r}_{1}\right)=-\frac{\delta n(\vec{r})}{\delta v_{x}\left(\vec{r}_{1}\right)}=-\frac{\delta^{2} P_{x}}{\delta v_{x}(\vec{r}) \delta v_{x}\left(\vec{r}_{1}\right)}
$$

and

$$
\begin{aligned}
s_{x}\left(\vec{r}, \vec{r}_{1}, \vec{r}_{2}\right) & =-\frac{\delta^{2} n(\vec{r})}{\delta v_{x}\left(\vec{r}_{1}\right) \delta v_{x}\left(\vec{r}_{2}\right)} \\
& =-\frac{\delta^{3} P_{x}}{\delta v_{x}(\vec{r}) \delta v_{x}\left(\vec{r}_{1}\right) \delta v_{x}\left(\vec{r}_{2}\right)}
\end{aligned}
$$

are exchange softness kernels.

\section{THE THOMAS-FERMI AND THE THOMAS-FERMI-DIRAC MODELS}

Let us suppose that the density is simply a function of $\widetilde{u}=v_{\mathrm{KS}}-\mu$; i.e., $n=n(\tilde{u})$. The hierarchy equations for the noninteracting kinetic energy will then lead to the Thomas-Fermi [11] approximation. If we use the expression

$$
\frac{\delta n(\vec{r})}{\delta \tilde{u}\left(\vec{r}_{1}\right)}=\frac{d n}{d \tilde{u}} \delta\left(\vec{r}, \vec{r}_{1}\right)=-s_{Q}\left(\vec{r}, \vec{r}_{1}\right),
$$

the first equation of the hierarchy, Eq. (47), takes the form

$3 n+\vec{r} \cdot \vec{\nabla} n=-\frac{d n}{d \tilde{u}} \widetilde{u}(\vec{r})+\frac{d n}{d \tilde{u}} \vec{\nabla}(\vec{r} \tilde{u})=2 \widetilde{u} \frac{d n}{d \tilde{u}}+\vec{r} \cdot \vec{\nabla} n$,

from which we obtain the equation

$$
\frac{d \tilde{u}}{\tilde{u}}=\frac{2}{3} \frac{d n}{n} .
$$

The solution is

$$
\tilde{u}=c n^{2 / 3} \text {. }
$$

Comparing it with the Euler equation of the ThomasFermi model

$$
\frac{5}{3} C_{\mathrm{TF}} n^{2 / 3}+\bar{v}=\mu,
$$

where

$$
\begin{aligned}
& C_{\mathrm{TF}}=\frac{3}{10}\left(3 \pi^{2}\right)^{2 / 3}, \\
& \bar{v}=-\frac{Z}{r}+\int \frac{n(\vec{r})}{\left|r-r^{\prime}\right|} d \vec{r}^{\prime}
\end{aligned}
$$

and

$$
\tilde{u}=\bar{v}-\mu,
$$

we see that this approximation is equivalent to the Thomas-Fermi model.

Similarly, with the assumption that the density is a function of the exchange potential

$$
n=n\left(v_{x}\right) \text {, }
$$

the first equation, Eq. (63), of the hierarchy for the exchange energy has the form

$$
\begin{aligned}
3 n+\vec{r} \cdot \vec{\nabla} n & =-2 \frac{d n}{d v_{x}} v_{x}(\vec{r})+\frac{d n}{d v_{x}} \vec{\nabla}\left(\vec{r} v_{x}\right) \\
& =v_{x} \frac{d n}{d v_{x}}+\vec{r} \cdot \vec{\nabla} n
\end{aligned}
$$

Equation (76) leads to the equation

$$
\frac{d v_{x}}{v_{x}}=\frac{1}{3} \frac{d n}{n},
$$

the solution of which is

$$
v_{x}=C_{x} n^{1 / 3}
$$

the well-known Dirac [12], Slater [13], Gáspár [14], and Kohn-Sham [15] expression for the exchange potential.

\section{AN ILLUSTRATION: THE PAULI POTENTIAL AND ENERGY}

As an illustration, we study the zeroth equation of the hierarchy for the Legendre transform of the noninteracting kinetic energy. The noninteracting kinetic energy $T_{s}$ can be written as a sum of the Weizsäcker $T_{w}$ and the Pauli $T_{p}$ energies,

$$
T_{s}=T_{w}+T_{p}
$$

where

$$
T_{w}=\frac{1}{8} \int \frac{|\vec{\nabla} n|^{2}}{n} d \vec{r} .
$$

The functional derivative of $T_{w}$ has the form

$$
\frac{\delta T_{w}}{\delta n}=\frac{1}{8}\left|\frac{\vec{\nabla} n}{n}\right|^{2}-\frac{1}{4} \frac{\nabla^{2} n}{n},
$$

and

$$
\frac{\delta T_{p}}{\delta n}=v_{p}
$$

is the Pauli potential. The Pauli energy and potential are the focus of research in the density-functional theory. The name derives from the fact that the appearance of the Pauli potential and energy is closely related to the Pauli principle. In an early study of the Weizsäcker functional, March and Murray [16] approximated the Pauli potential as

$$
v_{p}=\frac{5}{3} C_{\mathrm{TF}} n^{2 / 3} .
$$

Since then, several studies have been published on this subject (see, e.g., [16-26]).

The Legendre transform of the noninteracting kinetic energy can also be separated into two terms,

$$
Q_{s}=Q_{w}+Q_{p},
$$

where 


$$
Q_{w}=T_{w}-\int d \vec{r} n(\vec{r}) \frac{\delta T_{w}}{\delta n}
$$

and

$$
Q_{p}=T_{p}-\int d \vec{r} n(\vec{r}) \frac{\delta T_{p}}{\delta n} .
$$

From the expressions of $T_{w}$ and $\delta T_{w} / \delta n$ one can check that

$$
T_{w}=\int d \vec{r} n(\vec{r}) \frac{\delta T_{w}}{\delta n}
$$

and

$$
Q_{w}=0 \text {. }
$$

So

$$
Q_{s}=Q_{p}=T_{p}-\int d \vec{r} n(\vec{r}) \frac{\delta T_{p}}{\delta n} .
$$

As the Legendre transform of the Weizsäcker term disappears, the quantity $Q_{s}$ has contributions only from the Pauli energy. Thus it is especially useful for studying the Pauli energy. From Eq. (87) we see that the Weizsäcker energy is a homogeneous functional of degree 1 in $n$. By definition [1], a functional $R[n]$ is a homogeneous functional of degree $k$ in $n$ if

$$
k R[n]=\int d \vec{r} n(\vec{r}) \frac{\delta R[n]}{\delta n} .
$$

For the Legendre transform $S$ of the homogeneous functional $R$,

$S=R-\int d \vec{r} n(\vec{r}) \frac{\delta R}{\delta n}=(1-k) R=\frac{1-k}{k} \int d \vec{r} n(\vec{r}) \frac{\delta R}{\delta n}$.

Thus $S$ is a homogeneous functional of degree $k /(1-k)$ in $\delta R / \delta n$. We may ask whether the Pauli energy $T_{p}$ and its Legendre transform $Q_{p}$ are also homogeneous functionals, i.e., whether the following relations hold:

$$
k T_{p}=\int d \vec{r} n(\vec{r}) v_{p}(\vec{r})
$$

or

$$
Q_{p}=(1-k) T_{p}=\frac{1-k}{k} \int d \vec{r} n(\vec{r}) v_{p}(\vec{r}) .
$$

In order to check the validity of Eqs. (92) and (93), a knowledge of the Pauli potential $v_{p}$ is needed. In a recent paper [23] I proposed a method for the calculation of the exact Pauli potential in the exchange-only density functional theory. Using the results of this method, the Pauli potential and the integrals in Eqs. (92) and (93) have been calculated for a number of atoms. The calculated "homogeneity factor"

$$
k=1-\frac{Q_{p}}{T_{p}}
$$

is presented in Table I. These results obviously show that the Pauli energy is not a homogeneous functional because
TABLE I. "Homogeneity parameter" for the Pauli energy in neutral atoms.

\begin{tabular}{rc}
\hline \hline$Z$ & $k$ \\
\hline 4 & 9.39 \\
5 & 6.19 \\
6 & 4.56 \\
7 & 3.71 \\
8 & 3.22 \\
9 & 2.88 \\
10 & 2.63 \\
11 & 2.67 \\
12 & 2.61 \\
13 & 2.58 \\
14 & 2.53 \\
15 & 2.48 \\
16 & 2.44 \\
17 & 2.40 \\
18 & 2.33 \\
\hline \hline
\end{tabular}

the factor $k$ is different for the different atoms. We mention in passing that the Thomas-Fermi-like approximation to the Pauli energy proposed by March and Murray [16] is a homogeneous functional with $k=\frac{5}{3}$. [Compare Eqs. (82), (83), and (92).] An interesting question is whether $\lim _{Z \rightarrow \infty} k=\frac{5}{3}$. We emphasize that in the calculation of $k$ the exact Pauli potential was used without any locality assumption. Recently Zhao, Morrison, and Parr [27] have made a similar study for the exchange.

\section{WEIGHTED-DENSITY AND GRADIENT APPROXIMATIONS}

As another illustration, the Legendre transform of the exchange energy is discussed in the weighted-density and gradient approximations. In the weighted-density approximation (WDA), $P\left[v_{x}\right]$ is expressed as

$$
P_{x}^{\mathrm{WDA}}\left[v_{x}\right]=\int d \vec{r} g\left(\mathbf{r}, v_{x}(\mathbf{r})\right) ;
$$

i.e., there is an explicit dependence of $g$ on the position variable $\vec{r}$ in addition to the dependence $n(\vec{r})$. The derivation of $P_{x}^{\mathrm{WDA}}$ gives the electron density

$$
n=-\left.\frac{\partial g}{\partial v_{x}}\right|_{r} .
$$

The first equation of the hierarchy, Eq. (63), takes the form

$$
3 n+\vec{r} \cdot \vec{\nabla} n=-\left.\left[\vec{r} \cdot \vec{\nabla} v_{x}+v_{x}\right] \frac{\partial^{2} g}{\partial v_{x}^{2}}\right|_{r} .
$$

Combining Eqs. (96) and (97), we obtain an equation for the electron density:

$$
3 n+\left.\vec{r} \cdot \vec{\nabla} n\right|_{v_{x}}=\left.v_{x} \frac{\partial n}{\partial v_{x}}\right|_{r} .
$$

In spherically symmetric systems it has the form 


$$
3 n+\left.r \frac{\partial n}{\partial r}\right|_{v_{x}}=\left.v_{x} \frac{\partial n}{\partial v_{x}}\right|_{r} .
$$

In the weighted-density approximation, Eq. (99) should hold. This equation can be helpful in constructing the explicit form of weighted-density approximations.

In the gradient approximation, the electron density can be written in the form

$$
n\left[v_{x}\right]=n\left(v_{x}, \vec{\nabla} v_{x}\right) .
$$

The first equation of the hierarchy, Eq. (63), leads to

$$
3 n=v_{x} \frac{\partial n}{\partial v_{x}}+2 \sum_{i} \frac{\partial v_{x}}{\partial x_{i}} \frac{\partial n}{\partial \frac{\partial v_{x}}{\partial x_{i}}}
$$

If $n$ depends only on $v_{x}(\vec{r})$ and $\left|\vec{\nabla} v_{x}(\vec{r})\right|$, Eq. (101) reads

$$
3 n=v_{x} \frac{\partial n}{\partial v_{x}}+2\left|\vec{\nabla} v_{x}\right| \frac{\partial n}{\partial\left|\vec{\nabla} v_{x}\right|}
$$

This expression is valid for any approximation for the density of the type in Eq. (100). It can be used to construct a new form of gradient approximation for the density.

Both expressions (99) and (102) provide the density as a function of the exchange potential and $r$ or $\left|\vec{\nabla} v_{x}\right|$. In the usual weighted-density and gradient approximations, however, the exchange potential is expressed with the density. This difference is not necessarily a shortcoming of this approach. It might be that it is easier to approximate the density than the exchange potential.

\section{APPROXIMATE FUNCTIONALS AND THE TRUNCATION OF HIERARCHIES}

The hierarchies of equations are exact. They hold for the exact functionals. Approximate forms of the functionals generally do not, or only approximately, satisfy these equations - but not always. Approximate functionals can exactly satisfy the hierarchy equations. For instance, in Sec. VII the Thomas-Fermi and the ThomasFermi-Dirac models are derived from the hierarchy equations for the noninteracting kinetic and exchange energies. The derivation is based on the locality assumption. If the functionals are local, the Thomas-Fermi-Dirac functionals are exact solutions. Furthermore, these are the only solutions; i.e., the hierarchy equations do not exactly hold for other local approximations.

Another example is the truncation of the equations of hierarchy. If we assume that the $(k+1)$ th and higher functional derivative of the functional $Q_{s}$ is zero; i.e., that the hierarchy is truncated to the $k$ th order, the solution is

$$
Q_{s}=B_{k}\left\{\int \tilde{u}(\vec{r}) r^{-(1+2 / k)} d \vec{r}\right\}^{k},
$$

where $B_{k}$ are constants. Equation (103) can be easily verified by substituting into Eqs. (47), (48), and the higher-order equations. Similarly, the truncation of the hierarchy of equations for the functional $P_{x}$ leads to the following expression:

$$
P_{x}=D_{k}\left\{\int v_{x}(\vec{r}) r^{-(2+1 / k)} d \vec{r}\right\}^{k},
$$

where $D_{k}$ are constants. The validity of Eq. (104) follows from Eqs. (63), (64), and the higher-order equations for the functional $P_{x}$. Then approximate functionals for $Q_{s}$ and $P_{x}$ are exact solutions of the hierarchy equations.

In the weighted-density and gradient approximations, Eqs. (95) and (100) were assumed and equations for the density were derived from the first equation of the hierarchy. These relations must be satisfied by the approximate functionals of Eqs. (95) and (100), so they can be helpful in constructing new approximate functionals.

In all examples an additional assumption was done. This assumption includes the approximation. To judge the quality of the approximation, the hierarchy can no longer be used; the validity of the assumption has to be studied by other methods.

\section{DISCUSSION}

The physical meaning of the functionals $Q_{s}$ and $P_{x}$ can be easily seen. By multiplying the Kohn-Sham equations

$$
-\frac{1}{2} \nabla^{2} \varphi_{i}+v_{\mathrm{KS}} \varphi_{i}=\varepsilon_{i} \varphi_{i}
$$

by $\varphi_{i}^{*}$, integrating, and summing for all the spin orbitals $\varphi_{i}$, we obtain

$$
T_{s}+\int n(\vec{r}) v_{\mathrm{KS}}(\vec{r}) d \vec{r}=E_{s},
$$

where

$$
E_{s}=\sum_{i} \varepsilon_{i}
$$

is the sum of the one-electron energies $\varepsilon_{i}$. With Eqs. (80) and (81), the Legendre transform of $T_{s}$ can be given by

$$
Q_{s}=E_{s}-N \mu \text {. }
$$

Similarly, the Legendre transform of $E_{x}$ can be obtained. Writing the total energy $E$ as

$$
E=T_{s}+J+\int n(\vec{r}) v(\vec{r}) d \vec{r}+E_{\mathrm{xc}}
$$

and using Eqs. (52) and (106), we get

$$
P=E-E_{s}+J,
$$

where

$$
J=\frac{1}{2} \int \frac{n\left(\vec{r}_{1}\right) n\left(\vec{r}_{2}\right)}{\left|\vec{r}_{1}-\vec{r}_{3}\right|} d \vec{r}_{1} d \vec{r}_{2}
$$

is the Coulomb repulsion energy.

In the Thomas-Fermi and the Thomas-Fermi-Dirac models, the physical meaning of $Q_{s}$ and $P_{x}$ can also be easily seen. Writing the Euler-Lagrange equation

$$
\frac{5}{3} C_{\mathrm{TF}} n^{2 / 3}+\bar{v}=\mu
$$

we obtain

$$
\widetilde{u}=\bar{v}-\mu=-\frac{5}{3} C_{\mathrm{TF}} n^{2 / 3},
$$


and Eqs. (42), (83), and (84) lead to

$$
Q_{s}=-\frac{2}{3} C_{\mathrm{TF}} \int n^{5 / 3} d r=-\frac{2}{3} T_{s} .
$$

With the exchange energy

$$
E_{x}=\frac{3}{4} C_{x} \int n^{5 / 3} d \vec{r}
$$

and the exchange potential, Eq. (78), we arrive at

$$
P_{x}=-\frac{1}{3} E_{x} \text {. }
$$

The electron density $n(\vec{r})$ is the fundamental quantity of the density-functional theory of many-body systems. That is why equations for the electron density are of outstanding importance. The hierarchy of equations for the noninteracting kinetic energy and the exchange energy is especially interesting because these equations are selfcontained; i.e., they contain only the electron density and its functional derivatives. The first equation of the hierarchy for these functionals provides interesting equations for the electron density. These are generally complicated integro-differential equations. However, in certain approximations they may be much simpler. For instance, in the weighted-density and the gradient approximations, the first equations of the hierarchy reduce to differential equations for the electron density. These equations may help in the construction of new approximations.

\section{ACKNOWLEDGMENTS}

This paper is based on work sponsored by the Hungarian-U.S. Science and Technology Joint Fund in cooperation with the National Science Foundation and the Hungarian Academy of Sciences under Project No. $146 / 91$. This work has also been supported by the OTKA Grant No. 1733/191, and the Hungarian Ministry of Education Grants No. 212/92 and No. 56/92.
[1] R. G. Parr and W. Yang, Density-Functional Theory of Atoms and Molecules (Oxford University Press, New York, 1989).

[2] M. Levy, Int. J. Quantum Chem. Suppl. 23, 617 (1989); in Perspectives in Quantum Chemistry, edited by J. Jortner and B. Pullman (Kluwer, Dordrecht, 1989); Phys. Rev. A 43, 4637 (1991); A. Görling and M. Levy, ibid. 45, 1509 (1992).

[3] Á. Nagy, Phys. Rev. A 47, 2715 (1993).

[4] M. Berkowitz, S. K. Ghosh, and R. G. Parr, J. Am. Chem. Soc. 107, 6811 (1983).

[5] M. Berkowitz and R. G. Parr, J. Chem. Phys. 88, 2553 (1988).

[6] R. G. Parr and W. Yang, J. Am. Chem. Soc. 106, 4049 (1984).

[7] W. Yang, R. G. Parr, and R. Pucci, J. Chem. Phys. 81, 2862 (1984).

[8] M. Levy and J. P. Perdew, Phys. Rev. A 32, 2010 (1985).

[9] R. G. Parr and J. L. Gázquez, J. Phys. Chem. 97, 3939 (1993).

[10] A. A. Kugler, Phys. Rev. A 41, 3489 (1990).

[11] L. H. Thomas, Proc. Cambridge Philos. Soc. 23, 542 (1927); E. Fermi, Z. Phys. 48, 73 (1928).

[12] P. A. M. Dirac, Proc. Cambridge Philos. Soc. 26, 376
(1930).

[13] J. C. Slater, Phys. Rev. 81, 385 (1951).

[14] R. Gáspár, Acta Phys. Acad. Sci. Hung. 3, 263 (1954).

[15] W. Kohn and L. J. Sham, Phys. Rev. A 140, 1133 (1965).

[16] N. H. March and A. M. Murray, Proc. R. Soc. London, Ser. A 256, 400 (1960).

[17] B. M. Deb and S. K. Ghosh, Int. J. Quantum Chem. 23, 1 (1983).

[18] N. H. March, Phys. Lett. 113A, 476 (1986).

[19] M. Levy and H. Ou-Yang, Phys. Rev. A 38, 625 (1988).

[20] R. F. Nalewajski and P. M. Kozlowski, Acta Phys. Pol. A74, 287 (1988); A. Nalewajski, ibid. A77, 449 (1990).

[21] Á. Nagy and N. H. March, Phys. Chem. Liq. 22, 129 (1990).

[22] Á. Nagy and N. H. March, Int. J. Quantum Chem. 39, 615 (1991).

[23] Á. Nagy, Acta Phys. Acad. Sci. Hung. 70, 33 (1991).

[24] A. Holas and N. H. March, Phys. Rev. A 44, 5521 (1991).

[25] Á. Nagy and N. H. March, Phys. Chem. Liq. 25, 37 (1992).

[26] J. A. Flores and J. Keller, Phys. Rev. A 45, 6259 (1992).

[27] Q. Zhao, R. C. Morrison, and R. G. Parr, Phys. Rev. A 50, 2138 (1994). 